\title{
Organisation perverse ou le pacte avec le Diable
}

\author{
Emmanuel Gratton $\star$ \\ LUNAM Université - Université d'Angers LPPL - UPRES EA 4638, Angers, França
}

\section{RÉSUMÉN}

Il est incertain de caractériser une personnalité perverse, de la définir comme structure. Il peut sembler encore plus audacieux de considérer ungroupe comme pervers et a fortiori de l'étendre à une organisation, sauf à identifier les fonctionnements récurrents qui s'apparentent à la perversion: utilisation d'autrui, retournement, négation de l'altérité.Comment situer la limite entre pathologie et déviance, entre ce qui relève de la structure ou de la relation, de l'individuel ou du collectif? Autant de questions qui trouveront quelques éléments de réponses dans l'analyse de la genèse et de l'évolution d'une organisation à fonctionnement charismatique.

Mots clé: perversion; organisation; pacte dénégatif; alliances inconscientes.

\section{Perverse Organization OR NASTY PaCt WITH The DEVIL}

\begin{abstract}
It is uncertain to characterize a perverse personality, defined as structure. It may seem even more daring to consider a group as perverse and a fortiori to extend to an organization. Some recurring operations are related to this perversion: instrumentality of others, reversal, negation of otherness. How to draw the line between pathology or deviance, between what is the structure or the relationship, between what is individual or collective? These questions find some answers in the analysis of the genesis and evolution of an organization with a charismatic leader.
\end{abstract}

Keywords: perversion; organization; denegatif pact; unconscious alliances.

\footnotetext{
^ Psychologue, Sociologue clinicien, Maître de conférences, L'UNAM (L’Université Nantes Angers Le Mans) - Université d'Angers LPPL - Laboratoire de Psychologie des Pays de la Loire (UPRES EA 4638). Adresse: Université d'Angers - Maison des Sciences Humaines. 5 bis Boulevard Lavoisier - 49045 Angers Cedex 1 (France).

E-mail: emmanuel.gratton@univangers.fr / emmanuel.gratton@numericable.fr
} 
Il est banal qu'en cas de crise apparaissent des divisions entre membres d'un même groupe. La crise, en effet, comme son étymologie nous l'indique vient signifier ce qui sépare. Elle révèle en fait l'écart entre la fiction et la réalité, l'idéal et les faits. Depuis Anzieu (1975) nous savons que tout groupe est provisoirement bercé par une illusion dite groupale comparable à ce que le rêve représente pour un sujet. Un groupe "rêve" ensemble. La crise sonne alors comme un réveil. Elle rompt le charme de nos relations imaginaires.

Ces crises semblent cependant prendre aujourd'hui des formes particulières et provoquées des conduites dont nous nous interrogeons sur la dimension perverse, (manipulation, mensonge, harcèlement...) entre membres au sein des organisations ou bien des dysfonctionnements institutionnels que nous pourrions aussi qualifier au niveau groupal de pervers. Gilles Herreros (2009) parle de La violence ordinaire dans les organisations qu'il distingue de la violence physique ou de la violence perverse. Pour lui la banalité de cette violence et l'indifférence qui l'entoure sont les causes du malaise grandissant et des crises que traversent les individus dans les organisations et produisent tant de souffrances au travail. Eugène Enriquez (2004, p. 50) interroge nos sociétés hypermodernes qui favoriseraient "la généralisation des conduites perverses dans nos sociétés". Il nuance cependant son propos en montrant que "nos sociétés ne "produiraient' pas seulement des individus adoptant des conduites perverses, elle produiraient également des individus ayant des comportements paranoïaques et ou mégalomaniaques" (ENRIQUEZ, 2004, p. 56), lesquels ne seraient d'ailleurs pas réductibles les uns aux autres. Roland Gori (2013) dans La fabrique des imposteurs postule que l'imposture est finalement une solution, solution qu'adoptent les individus pour leur fonctionnement psychique face aux changements majeurs de notre civilisation, ce que Jean-Pierre Lebrun avait déjà identifié comme "perversion ordinaire". André Sirota (2003, p. 226) va encore plus loin en présentant les différentes Figures de la perversion sociale:

On est fondé à penser qu'un individu normal, non particulièrement disposé, $\mathrm{du}$ fait d'une structure psychopathologique, à devenir un pervers psychosocial, immoral, peut le devenir dans certaines conditions politiques d'existence, du fait des structures sociopathiques de la société.

Les liens entre perversions narcissiques et perversions sociales sont donc à établir et je vais tenter de m'appuyer sur une organisation dans laquelle je suis intervenucomme psychosociologue pendant trois ans pour en démêler les nœuds bien que d'autres processus non pervers peuvent y être aussi associés.

\section{LE SYSTÈME D'UNE ASSOCIATION SYSTÉMIQUE}

Les membres de cette association d'approche systémique partagent imaginairement le même projet, le même idéal. Cette association repose sur un engagement équivalent de chacun de ses membres, censés être non intéressés à titre individuel, et reposant sur un idéal égalitaire. La structure existe depuis une quinzaine d'années et a pour objet de former des praticiens à la systémie. Elle est cen- 
trée autour d'un leader charismatique, cofondateur de la structure et est dirigée par un des membres. Des commissions sont chargées de certaines missionsspécifiques afférentes à la communication ou à l'organisation de manifestations.

Le groupe a connu dans son avènement des crises relationnelles auxquelles je n'ai pas assistées et dont je n'ai eu qu'un écho. Il me semble difficile ici de rapporter des éléments qui tiennent simplement au discours véhiculé et à leur interprétation à l'aune d'événements consécutifs. Ils procèdent d'une "relecture", d'une mise en récit qui prend soudainement un sens collectif chez certains membres là où ils n'apparaissaient jusqu'alors que comme des positions individuelles. La société a été remaniée il y a 7 ans environ avec l'arrivée de nouveaux membres dont celle du directeur actuel. La structure traversait déjà des difficultés financières et le nouveau directeur apparaissait alors comme le sauveur de la structure en offrant au-delà de sa seule fonction, les moyens humains et matériels de son réseau pour assurer le secrétariat et les locaux nécessaires à l'activité de formation.

Je commence à intervenir à raison d'une fois par mois alors que de nouveaux membres aspirent à entrer dans ce cercle. Le protocole pour entrer dans cette structure nécessite de faire ses preuves aussi bien sur le plan intellectuel que morale. Il s'agit de partager la philosophie de la dite structure et de faire preuve des compétences adéquates. Certains rituels institutionnels, examens et épreuves sont organisés afin de permettre l'intronisation de nouveaux membres.

Une crise financière surgit également au moment même où de nouveaux membres demandent à entrer dans la structure. Cette nouvelle crise va cristalliser des oppositions fortes entre ces membres créant ainsi la formation progressive de deux pactes. L'opposition va créer un climat de suspicion sur l'origine de la crise financière notamment envers le directeur, lequel est appuyé par le leader charismatique. Alors qu'une sorte de pacte mystérieux semble les associer, d'autres pactes entre les autres membres se dessinent aussi.

La crise se traduit par des invectives, accusations et insultes entre membres, par la disqualification des instances électives prévues par l'organisation La seconde se traduit par la recherche d'alliances pour refonder la société. $\mathrm{Au}$ final se dessine une sorte de pacte dénégatif agi par des forces inconscientes qui conduisent à l'éclatement et la division de l'association. Dans cette lutte, quel usage a été fait finalement de la perversion?

\section{LA FORMATION DE DEUX PACTES ET D’UNE MÊLÉE}

\section{A. Le point d'origine}

On sait que les groupes achoppent régulièrement sur des questions de transmission ou d'élargissement. Les "impétrants" suscitaient des divisions entre membres. La cooptation de chacun n'était pas unanime. L'association décida la mise en place d'un jury, lequel était composé de quatre membres dont le directeur. Le jury a refusé la candidature d'une "prétendante" laquelle était "la préférée", du chef charismatique, celle qui se situait selon lui au dessus des qualités intellectuelles et morales de tous les autres. Cette disqualification a provoqué une 
scission au sein de l'institution et a empêché pendant un an environ le fonctionnement normal de l'institution. Le conflit de pouvoir entre le leader charismatique et certains membres de cette commission dont en particulier le directeur et une autre adhérente que je nommerai "la rebelle". Cependant le directeur, las de ces tensions et sous la pression du chef charismatique, a finalement "retourné sa veste" pour "la belle", trahissant ainsi "la rebelle".

Cet épisode a laissé des traces indélébiles sur le fonctionnement de la structure et il s'est formé progressivement deux pactes, l'un autour de la légitimité du leader et de sa position, l'autre autour des forces de contestations incarnées par la rebelle. Ces tensions ont tenté d'être régulées au sein du groupe une journée entière, journée dont j'avais la charge de l'animation. Chacun s'est exprimé et un pacte de paix s'est organisé autour de la reconnaissance officielle de l'entrée comme membre de "la belle". Cet épisode m'a permis de tirer certaines conclusions sur la faible crédibilité du directeur que j'avais entendu argumenté longtemps sur l'incompétence de "la belle" et sur les pressions répétées exercées contre lui par le chef charismatique. Au niveau de chaque membre de l'association, il fallait gérer les conflits intrapsychiques et conflits interpersonnels, la résolution de l'un provoquant l'apparition des autres. C'est en fait à un phénomène de désillusion groupale auquel on pouvait assister.

\section{B. D'un déficit financier à un déficit d'estime}

A cette crise de confiance est venue s'ajouter une crise financière, à l'image de celle qui traverse aujourd'hui notre société malade de la gestion(GAULEJAC, 2005 ) et qui révèle chaque jour "les petits arrangements" entre acteurs consentants. La gestion a fait soudainement apparaitre un déficit. Le dit déficit s'est accentué alors que les activités de la structure étaient en progression.Les causes de ce déficit étaient imputées par le directeur à certaines activités spécifiques du leader charismatique, lesquelles généraient selon lui des taxes collectives importantes. Certains membres ont décidé de recourir à un audit externe pour connaître les causes réelles du déficit, audit auquel se sont opposés le directeur et le leader charismatique.

Les pactes qui étaient alors en préfiguration se sont plus nettement dessinés. Les personnes en position médiatrices ont du choisir leur camp. Huit membres sur seize ont donc décidé de manière indépendante de faire appel à un avocat pour clarifier si les causes du déficit étaient imputables aux activités spécifiques du leader charismatique et pour établir un diagnostic sur le mode de gouvernance. Le directeur a fini une nouvelle fois pas conclure que le déficit n'était pas imputable aux activités spécifiques du leader charismatique, retournant une nouvelle fois sa veste.

\section{La systémie dans la mêlée}

Dans le camps des opposants, il y avait la rebelle mais aussi tous ceux qui à un titre ou un autre pouvaient avoir des comptes à régler avec le chef charismatique et/ou le directeur. Dans le camp des alliés, il y avait outre le leader et le directeur, ceux qui avaient des relations privilégiés avec eux et/ou un intérêt affectif ou de carrière à se situer à leurs côtés. A chaque poussée adverse, l'obligation de la poussée s'imposait. S'il fallait caractériser les deux clans, les 
deux blocs, il y avait les justiciers, ceux qui a un titre ou à un autre avaient le sentiment d'avoir été lésé et/ou blousé par les dirigeants masqués et d'un autre, les défenseurs de la légitimité des puissants qui prônaient l'égal responsabilité de tous les membres. Les uns dominés étaient pris pour des persécuteurs, les autres dominants se présentaient comme les persécutés. Mais dans la mêlée, tout le monde est gris. Cette situation frontale faisait ressurgir toutes les médisances des membres du camp adverse. Il apparaissait alors un autre monde que celui qui avait permis le rassemblement premier. Les rapports de pouvoir, de sexe, d'argent, de dépendance entre membres étaient dévoilés au détriment des relations relatives au projet institutionnel. La charte des membres disparaissait par exemple dans la mêlée. Ce clivage mettait aussi en difficultés les relations d'amitié entre membres des deux camps. Les relations devenaient fausses: fausse naïveté, mauvaise foi, mensonges, fausses allégations, dénigrement, interprétation sauvage, accusation, procès d'intention.... Les mauvais coups étaient donc aussi au rendez-vous. La perversion y jouait-elleun rôle?

La formation en pactes est une conséquence du jeu pervers qui fait de la force la loi et non plus de la loi, la force.

Dans toute situation de conflit ou de compétition, La victoire peut s'obtenir de deux façons. Ou bien parce qu'on est le plus fort sur le terrain en question. Ou bien par l'utilisation de procédés qui ont pour but de fausser l'épreuve et de faire triompher celui qu'on croyait battu. On peut considérer la mètis comme ce qui amène la fraude ou, au contraire, comme ce qui crée la surprise et la revanche du plus faible. (VIGNAUX, 2001, p. 9).

Ce jeu s'est particulièrement mis en place lorsqu'il s'est dessiné l'appel à un tiers externe, en l'occurrence une avocate pour dire la loi, une loi susceptible de s'élever au dessus de la mêlée. La loi a été absorbée par le jeu et non plus le jeu dirigé par la loi. L'avocate comme symbole est entrée dans la mêlée. Elle est devenue l'avocate des huit et non plus la loi universelle. Sans ballon, ni arbitre, la mêlée a continué de pousser et comme cela arrive souvent au rugby, elle a perdu toute cohésion. Les coups, les coups bas ont commencé à pleuvoir. Il y a eu parfois des tentatives de diversion pour atténuer la force adverse, puis des coups de force ou des coups de ruse.

Les différentes crises successives qui ont traversées l'association renvoient pour la plupart à son fondement et sont particulièrement actualisés par son risque objectif de disparition (déficit) ou par sa transformation profonde (arrivée des nouveauxmembres).

\section{Le PaCte des frères P.}

Il y a dans toute histoire groupale une origine, un mythe originaire. En ce qui concerne cette structure, l'origine de ce mythe peut varier selon les versions et selon la date d'engagement des intéressés. Faire l'histoire de cette association 
a été réalisée par un membre mais s'agit-il d'un travail "scientifique" d'historien ou d'une simple version de l'intime. Y a t-il une vérité, une seule version de l'histoire, une père-version pourrait-on dire ou bien des vérités, différentes versions de l'histoire, des frères-versions par analogie, chacune de versions des frères prétendant à la version du père.

\section{A. Un pacte d'origine}

Le directeur et le leader charismatique ont une histoire commune très ancienne, qui échappe en partie aux autres membres. Ils ont eu le même père spirituel, ce qui en fait des frères. Comme Caïn et Abel, ils connaissent régulièrement des rivalités d'héritiers l'un ayant davantage opté pour le versant thérapeutique du père spirituel, l'autre pour son versant théorique. Comme des frères, ils semblent avoir scellés un pacte d'exclusivité sur l'héritage en question. Ils trouvent d'ailleurs une certaine complicité lorsqu'il s'agit de discourir sur les "pathologies" dites "systémiques", que ce soit sur un versant clinique ou que ce soit sur un versant social. Mais comme pour Caïn et Abel, ce pacte est suspendu à leur stratégie plus individuelle et le meurtre de l'un sur l'autre apparaît toujours comme possible. Cette potentialité meurtrière fait dissuasion car ils partagent probablement des secrets d'origine qui pourraient, réellement ou imaginairement, les anéantir.

\section{B. Un pacte d'intérêts croisés}

Le pacte repose aussi sur des intérêts croisés. L'un sert la cause de l'autre et réciproquement, le premier par la direction de la société et la possibilité de faire des affaires, l'autre par la notoriété scientifique et la caution qu'il donne à la structure. Il s'est installé entre eux comme un pacte mêlant leurs intérêts communs de telle sorte que l'un ne puisse se désolidariser de l'autre. Cependant leur relation, à l'examen, est plus complexe qu'il n'y paraît car ils peuvent aussi être adversaires: l'un dénonce l'incompétence du directeur, le second traite l'autre de "pervers narcissique". Cette notion de "pervers narcissique" traduit en fait une forme d'emprise qu' exerce le leader charismatique sur le directeur et dont ce dernier se sent probablement "le complice" (EIGUER, 2006). Alberto Eiguer (2006, p. 70-71) compte parmi les six traits du transfert pervers "le pacte ou contrat pervers" qu'il désigne comme:

la recherche d'un pacte secret avec l'analyste afin que celuici abandonne un ou plusieurs termes du contrat analytique en lui proposant, par exemple, une offre alléchante (séduction narcissique); et cela de façon défigurée et totalement cachée.

Dans le cas qui nous occupe et même si aucun n'est l'analyste de l'autre (quoi que l'on pourrait parler probablement d'intertransfert), il est probable que le leader ait menacé de manière masquée le directeur de la structure, s'il continuait de refuser la cooptation de "la belle", ce dernier ayant luiaussi une compagne qui candidatait pour son entrée. Cette réciprocité directe d'échange de "femmes" masquait probablement en fait un pacte plus obscur à caractère dénégatif et dont l'em- 
prise était certainement davantage mortifère. Le complice qui ne parvient pas à se déprendre du maître en s'opposant à lui cherche alors les moyens d'y parvenir en lui ressemblant. S'il ne peut l'égaler, il reste cependant encore à la merci du maître.

Mon analyse dans cette histoire est particulière car je ne pouvais pas les partager ouvertement. Le directeur, dans un moment de détente, me dit avoir rêvé de moi en Zorro, lequel peut être aussi bien pris pour un sauveur qu'un vengeur masqué. Il se joue dans cette partie de transferts projectifs place à tous les fantasmes. Qui est Bernardo me demandai-je?

\section{Un pacte secret}

Depuis le pacte du leader et du directeur, d'autres associés ont également été désignés de "pervers narcissiques" par le directeur, ceux là qui dénoncent précisément l'emprise dont il a fait l'objet par le maitre. Cette emprise l'a conduit à retourner maintes fois sa veste et ceci même après la tractation supposée. Les associés en voulant recourir à un audit lui rappelle indirectement l'emprise dont il a fait l'objet au moment où le pacte s'est scellé. Le directeur se trouve pris en quelque sorte en tenailles, entre l'aveu et le pacte. En attaquant "le complice", les associés attaquent aussi indirectement le maître et renforce le pacte passé entre eux, pacte qui maintient le secret des tractations conscientes et cachées et peut être de manière plus éloignée encore, le secret des origines et les revendications enviées dans l'héritage du père. "Clavreul a souligné l'importance du secret dans le contrat qui lie les deux partenaires du couple pervers. C'est la dénonciation du secret, la mise au courant de tiers, le scandale qui constitue la rupture" (KAES, 2009, p. 134).

Cette association de "frères ennemis" s'est manifestée par les renversements de position du directeur, d'une part à l'égard de "la belle" devenue soudainement "cooptable" et d'autre part à l'égard des activités spécifiques du leader, tout d'abord mises en cause et soudainement favorables à la structure. Il a alors tenté de déplacer la question des problèmes financiers sur les activités d'autres membres, ceux et celles qui l'attaquaient de front sur sa mauvaise gestion. Pour se défendre il avait alors besoin du secours du leader qui se trouvait lui aussi menacé par leurs "petits arrangements entre frères/amis".

\section{Un pacte pervers qui s'étend à l'organisation}

Face à autant d'adversité, ils réfutèrent l'un de manière ouverte et l'autre de manière masquée le recours à un tiers externe pour dire la loi car la loi, c'est eux qui sont censés l'incarner. Ils peuvent ainsi maintenir le pacte pervers qui les unit et auquel ils sont eux-mêmes aliénés.Comme le souligne Albeto Eiguer (2006, p. 70-71):

On observe plutôt chez le pervers narcissique une recherche désespérée pour éloigner le danger de la perte de son intégrité narcissique [...]Toutefois le pervers narcissique ne parvient jamais à éloigner définitivement sa crainte de la désintégration. Il doit alors perpétuer son jeu avec l'autre pour se rassurer lui-même sur sa puissance, la grandiosité de 
son self et sa capacité à réalimenter sans cesse les "batteries". Comme il n'est jamais réconforté et bien qu'il fasse beaucoup d'effort pour nier son attachement à son objet, il est tout aussi dépendant de cet objet que cet objet de lui.

André Sirota (2003, p. 49-50) décrit les buts poursuivis par les pervers psycho-sociaux :

- "Détourner l'attention de ses failles et stratagems"

- "Paralyser le groupe en inspirant à ses membres le sentiments qu'il sont nuls"

- "Accéder à la jubilation perverse"

- "Une manœuvre et un but défensif"

On notera que ces stratagèmes sont plus manifestes chez le directeur que chez le leader car chez ce dernier, ils sont plus invisibles. Il fait agir le directeur tout en se protégeant des retombées que cela pourrait avoir sur lui. J'ai reconnu par exemple un document dont une partie ne pouvait qu'avoir été écrite par le leader mais qui était signé du directeur.

La rivalité des frères $\mathrm{P}$. s'est manifestée à plusieurs reprises, dans l'opposition vaine du directeur à coopter "la belle", dans la dénonciation indirecte des activités spécifiques du leader. Mais le leader connaît les faiblesses de son directeur et a trouvé les moyens d'en faire un complice, comme il repère aussi les faiblesses d'autres membres pour en faire aussi des complices. La perversion est donc circulaire et si elle est initiée par ceux qui ont le pouvoir, elle traverse toute la société.

\section{UNE ORGANISATION PERVERSE?}

Le pacte des frères étendue à l'ensemble des membres se présentent ici comme une rivalité fraternelle : "Lorsque l'alliance totémique est menacée par l'émergence d'un clan qui parmi les Frères forment ligue pour renverser le Père et ses valeurs, alors se forme le pacte des Frères fidèles pour sauver le Père et son institution" (KAES, 2009, p. 83). Ce n'est pas un hasard si certains frères/soeurs doivent être éliminés au profit des fils et filles, futurs frères et sœurs dans la structure, car ces derniers ne peuvent que reconnaître la générosité de leur maître et accepter que la démocratie n'advienne que plus tard. Elles maintiennent le représentant du Père, le Frère leader, héritier et père à son tour, ayant pris place, et depuis longtemps, dans la direction du pouvoir. La perversion consiste ici à faire prendre la royauté pour la démocratie, l'adoubement pour l'habilitation, la loi du père pour la loi des frères. A la loi démocratique officielle où les frères sont égaux est maintenue une loi patriarcale et arbitraire dans laquelle une nouvelle génération efface les attributs conquis de l'ancienne génération. Cette passation de pouvoir, "cette réalimentation des batteries" entre générations nécessite le maintien de l'organisation actuelle par substitution des places et non redistribution élargie des pouvoirs et remise en cause, par exemple, des clés de répartition, celles ci pouvant être entendues de manière financière ou symbolique. 
$\mathrm{Au}$ pacte secret des frères $\mathrm{P}$. s'est substitué donc d'autres pactes entre membres qui n'en sont pas nécessairement moins pervers. Il règne alors une ambiance de complots, d'instrumentalisation, d' alliances inconscientes. Les alliances structurantes primaires ont laissés place à des alliances structurantes secondaires qui elles mêmes ont donné place à des alliances métadéfensives ou offensives. Les alliances offensives ont conduit à la formation de deux pactes offensifs comme nous l'avons vu.Les alliances métadéfensives se traduisent par des pactes dénégatifs (dénégation, déni, désaveu, rejet). Le pacte dénégatif est "un pacte sur le négatif". Ces pactes sont conclus "pour assurer les besoins défensifs des sujets lorsqu'ils forment un lien et pour maintenir ce lien" (KAES, 2009, p. 113). Ils se situent donc à la frontière de l'intra psychique et de l'intersubjectif. Le pacte dénégatif s'inscrit dans différentes formes de négativité, négativité d'obligation, négativité absolue ou relative. "Les opérations psychiques que suscite la négativité d'obligation ont pour but de préserver un intérêt majeur de l'organisation psychique, celle du sujet lui-même en celle des sujets auxquels il est lié par un intérêt majeur" (KAES, 2009, p. 105).

On retrouve chez les opposants au directeur et au leader les besoins d'existence et d'intégrité (obligations faite à soi-même). L'obligation est alors de rester liés pour éviter toute perte narcissique. Face à cette nécessité d'illusion groupale, les sous-groupes oscillaient entre négativité relative - l'incertitude est explorée sans danger grâce à la croyance dans le meilleurs des groupes possibles - et négativité absolue, la menace de destruction unifiant le groupe et ses membres. Cette obligation de solidarité entraine des formes de soumission entre membres au sein du clan. Ce dernier dénonçait les agissements du directeur, du leader et de ceux qui les soutiennent. Ce devoir de solidarité ne peut qu'entrainer des formes d'emprise également des uns et des autres, des pactes plus ou moins conscients, même si certains rappelaient le devoir d'éviter les processus claniques. Qui fait quoi? Qui est en première ligne et qui reste dans l'ombre? Certains membresmanifestaient des formes de dépression à tour de rôle et le soutien des autres membres permettait alors de relever la tête.

Pour autant, la répétition des fronts et des affronts mettaient régulièrement en danger les relations et provoquaient des attitudes fortement marquées d'ambivalence. Les membres adeptes pourtant d'approche systémiqueétaient meurtris par l'alliance d'autres amis. Le maintien des liens de certains membres de camps adverses s'opérait en évitant autant que faire ce peut d'être envahi des toxicités des processus groupaux. Certains ignoraient mes analyses ou feignaient d'ignorer les injures publiques du directeur à l'égard de certains membres. Une certaine répétitivité et chronicité des relations d'hostilité s'est donc installée. "Leurs effets se manifestent dans les répétitions et les symptômes partagés, dans les objets bizarres ou énigmatiques, dans les acting" (KAES, 2009, p. 120). 


\section{Conclusion}

Une telle histoire institutionnelle est d'une banalité affligeante. Elle interroge cependant les processus pervers qui peuvent se mettre en place. Ils le sont à un triple titre. Toute forme de relation à l'autre engage une forme d'exploitation perverse de l'autre pour satisfaire ses pulsions propres, que ce soit dans le champ de la sexualité (comme à son origine), de l'amitié, du travail... La perversion est donc l'affaire de tous car comme le dit Piera Aulagnier (1967, p. 79): "De la question perverse nous ne pourrons jamais dire qu'elle ne nous regarde pas, sûrs que nous sommes qu'elle, de toute façon, nous regarde". La perversion engage soit le sujet en tant qu'objet de l'autre ou son envers, en tant que négation de l'autre comme sujet. Le processus pervers caractérise, selon Joël Dor (1993, p. 317), les “sujets qui n'accepteront l'incidence de la castration que sous réserve de la transgresser continuellement".

Le deuxième niveau de la perversion se situe dans le champ organisationnel lui même, dans la lutte des places et des moyens utilisés pour en jouir. "Tout se passe comme si dans les perversions, le sujet parvenait à maintenir ce paradoxe psychique qui consiste à savoir quelque chose de la castration, tout en n'en voulant rien savoir" (DOR, 1993, p. 316). Cette contestation de la castration se situe ici dans l'aveuglement qui est opéré par, sur et entre le leader comme "mère phallique" et ses "sujets-objets" maintenus en position infantile. L'organisation, le Moi groupal est clivé comme peut l'être le moi dans le cas du déni de castration. Le directeur, celui qui gère, s'est construit lui aussi dans une forme de déni de la réalité, espérant pouvoir jouir infiniment de cet aveuglement en simulant, leurrant, travestissant la réalité.

Le troisième niveau se situe sur les effets produits chez chaque sujet du fait de la mise en place d'un tel clivage et nous touchons ici la perversion sociale proprement dite, celle qui consiste pour survivre à faire "pacte avec le diable", à renverser l'amour en haine de l'autre et à utiliser pour ce faire, tous les artifices de la réalité pour en jouir encore. La meilleure des copies d'un tableau de maître est celle qui ressemble le plus à la réalité mais elle est en même temps celle qui nous trompe davantage encore. Pour débusquer cette tromperie, il s'agit alors de devenir plus rusé et de démasquer le rusé en utilisant parfois les mêmes moyens. Faut il faire alors pacte avec le diable? 


\section{RÉFÉRENCES}

ANZIEU, D. Le groupe et l'inconscient. Paris : Dunod, 1975.

AULAGNIER, P. Remarques sur la féminité et ses avatars. In: AULAGNIER, P. et al. (Org.). Le désir et la perversion. Paris: Seuil, 1967. p. 55-89.

DOR, J. Perversion. In: KAUFMANN, P. (Dir.). L'apport freudien: éléments pour une encyclopédie de la psychanalyse.Paris: Bordas, 1993. p. 314-321.

EIGUER, A.Le pervers narcissique et son complice.Paris: Dunod, 1996.

ENRIQUEZ, E. L'idéal type de l'individu hypermoderne: l'individu pervers?.In: AUBERT, N. (Org.). L'individu hypermoderne.Ramonville Saint-Agne: ERES, 2004. p. 39-57.

GAULEJAC, V. de.La société malade de la gestion: idéologie gestionnaire, pouvoir managérial et harcèlement social.Paris: Seuil, 2005.

GORI, R.La fabrique des imposteurs.Paris: Les Liens qui Libèrent, 2013.

HERREROS, G.La violence ordinaire dans les organisations: plaidoyer pour des organisations réflexives.Ramonville Saint-Agne:ERES, 2009.

KAES, R.Les alliances inconscientes.Paris: Dunod, 2009.

SIROTA, A.Figures de la perversion sociale.Paris: EDK, 2003.

VIGNAUX, G. Les jeux des ruses: petit traité d'intelligence pratique.Paris: Seuil, 2001.

Reçu: Juillet 06, 2013 Accepté: Septembre 24, 2013 
